\title{
Corrigendum: Direct observation of dynamic charge stripes in $\mathrm{La}_{2-x} \mathrm{Sr}_{x} \mathrm{NiO}_{4}$
}

S. Anissimova, D. Parshall, G.D. Gu, K. Marty, M.D. Lumsden, Songxue Chi, J.A. Fernandez-Baca, D.L. Abernathy, D. Lamago, J.M. Tranquada \& D. Reznik

Nature Communications 5:3467 doi: 10.1038/ncomms4467 (2014); Published 17 Mar 2014; Updated 3 Jul 2014

One of the affiliations for D. Lamago was inadvertently omitted during the preparation of this Article, and is given below: Institute of Solid State Physics, Karlsruhe Institute of Technology, D-76021 Karlsruhe, Germany. 